\title{
A review on the effect of rubber membrane in triaxial tests
}

\begin{abstract}
This paper presents a review on the effect of rubber membranes on the measured stress and volume change data during the triaxial tests. In many instants, the error associated due to membrane penetration is measured to be close to the total sample volumetric strains. Hence, in addition to precise measurements, adopting an appropriate correction to the measured data is pivotal. This paper provides a detailed review on the methods used to estimate and reduce the influence of rubber membrane on the measured triaxial test results. The discussion therefore clearly highlights the stiffness, thickness, and diameter of the membrane used, to have significant influence on the measured deviatoric stress and the volume change data. The mean grain size of sample has an equally significant influence on the volume change. The methods in general are limited to test conditions, and therefore, it is practically uncertain to have a generalized correction procedure. Effectively, the outcome from this review work enhances the understanding of the reader towards the effect, methods, and corrections to be considered in triaxial testing due to rubber membrane and membrane penetration effects.
\end{abstract}

Keyword: Triaxial test; Rubber membrane; Membrane penetration; Deviatoric stress; Volumetric strains 